\title{
Integral transform approach for dynamic fracture calculation and crack growth modelling
}

\author{
P. Brož \\ Professional Association for Science, Research and Consultancy, \\ Czech Republic
}

\begin{abstract}
The analytical mould of the solution in the radial direction permits precision stress intensity factors to be derived in the scaled boundary finite element method, namely, directly from the definition, and consequently no particular crack-tip interpretation such as refining the crack-tip mesh or employing singular elements, is indispensable. Besides, anisotropic material characteristics can be treated effortlessly. In this analysis, the Frobenius approach in the frequency domain to the solution of the governing differential equations of the SBFEM is used to simulate variable dynamic fracture problems. The complex frequencyresponse functions are calculated. Thereafter, the dynamic stress intensity factors are squarely taken from the response functions out. That is ensued by a fast Fourier transform of the transient load and a later inverse transform to derive the time history of DSIFs.

A mixed-mode crack growth simulation was developed. At first, a domain is divided into some subdomains. Since the dimensions and shapes of subdomains can be flexibly changed and only the domain boundaries or common edges between subdomains are discretized in the SBFEM, a remeshing routine such as a straightforward one as in BEMs was set up with minimum mesh variances while the universality and flexibleness of the FEM is preserved.

Keywords: crack trajectory, frequency-domain approach, response function, internal damping coefficient, load-displacement relation, mesh density, remeshing routine, subdomain, transient fracture problem.
\end{abstract}




\section{Introduction}

For analyzing dynamic stress intensity factors, several numerical methods were evolved and used. As regards how the simulated domain is discretized in space, these techniques may be in general split up into four types: the finite difference method (FDM), the finite element method (FEM), the boundary element method (BEM); the meshless or meshfree method are more recently and extensively used in modelling mixed-mode crack growth. As regards how the time processes of DSIFs are calculated, the methods given can be arranged into time-domain ways based on the direct time integration, and integral transform methods inclusive of the Laplace transform and the Fourier one (that means, the frequency-domain method in virtue of frequency analysis, and the discrete or fast Fourier transform (FFT). Superb literature surveys of these methods were presented by Ariza and Dominguez [1], and Song [2].

A frequency-domain interpretation approach has certain benefits over the time-domain, such as no demand for a mass matrix, and so coarser meshes may be applied, and as soon as a complex frequency-response function is obtained, it can be employed in combination with FFT and inverse FFT (IFFT) to compute transient responses for different forms of dynamic loading.

The scaled boundary finite-element method (SBFEM), developed lately in $[3,4]$, is a semi-analytical technique joining the advantages of FEM and BEM, i.e., it discretizes boundaries merely so that the simulated spatial dimensions are made smaller by one like the BEM and meantime it does not require fundamental solutions as the FEM.

In consequence, the considerable usability of the FEM and the easiness in remeshing of the BEM can be potentially kept.

The modelling of mixed/mode crack propagation is accomplished by combining the remeshing routine with a growth criterion. The developed simulation is competent of predicting crack trajectories and load-displacements dependences precisely and effectively.

Model problems with isotropic and anisotropic material characteristics are simulated by applying the frequency-domain procedure. The influences of the internal damping coefficient, the mesh density, the frequency interval and the maximum frequency are debated as well. The DSIFs $K_{I}$ and $K_{I I}$ are extracted from the stress solution for a wide range of frequencies, resulting in complex frequency-DSIFs response functions. These functions are later applied to generate time histories of the DSIFs. This procedure is accurate and efficient with a small number of degrees of freedom.

In the paper, a frequency-domain technique is evolved for transient dynamic fracture analysis applying the SBFEM according to [5]. The Frobenius solution [6] developed in a different way in the frequency domain, is used to determine complex frequency-DSIFs functions that are afterwards employed with fast Fourier transform and inverse FFT to calculate the time process of DSIFs.

The static conception within the bounds of the SBFEM is presented, including instructions how to extract SIFs from the solution, in compliance with [6]. Considering the advantages of the SBFEM, a very straightforward remeshing 
routine is evolved, in company with a concise discussion of mixed-mode crack growth criterion and explanation of the scaling routine.

\section{Computation of dynamic stress intensity factors}

A cracked region simulated by the SBFEM is indicated in fig. 1. All the time, the scaling center is located at the crack tip. The square-root singularity existing at this tip in a homogenous plate are the circumstances very considerably studied. In this instance the mode-I and mode-II SIFs are defined being

$$
\left\{\begin{array}{l}
K_{I} \\
K_{I I}
\end{array}\right\}=\lim _{r \rightarrow 0}\left\{\begin{array}{l}
\left.\sqrt{2 \pi r} \sigma_{y y}\right|_{\theta=0} \\
\left.\sqrt{2 \pi r} \sigma_{x y}\right|_{\theta=0}
\end{array}\right\}
$$

where $r$ and $\theta$ are the polar coordinates with the origin at the crack tip (see fig.1).

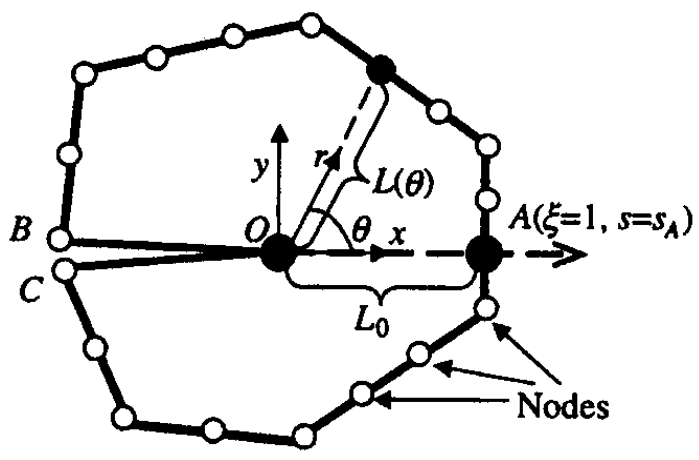

Figure 1: A cracked domain simulated by the scaled boundary finite-element method (taken from [5]).

Notice the relation between $r$ and $\xi(\xi=0$ at the crack tip and 1 at the boundary, according to fig. 1) has the form

$$
r=\xi L(\theta)
$$

where $L(\theta)$ is the distance between the crack tip and the intersection point of the polar line $r$ and the region boundary. In compliance with the Frobenius solution procedure outline and considering Eq. (1) we get

$$
\left\{\begin{array}{l}
K_{I} \\
K_{I I}
\end{array}\right\}=\lim _{r \rightarrow 0}\left\{\begin{array}{l}
\left.\sqrt{2 \pi L(\theta)} \sum_{i=1}^{n} c_{i} \xi^{\left(\lambda_{i}-0.5\right)} \psi_{y y}(s)_{i}\right|_{\theta=0} \\
\left.\sqrt{2 \pi L(\theta)} \sum_{i=1}^{n} c_{i} \xi^{\left(\lambda_{i}-0.5\right)} \psi_{x y}(s)_{i}\right|_{\theta=0}
\end{array}\right\}
$$

The stress field expression is a sign of all the stress modes with $\lambda_{\mathrm{i}} \geq 1$, disappears for $\xi \rightarrow \theta$ or $r \rightarrow 0$. Just two modes with $\lambda_{\mathrm{i}}=0.5$ results in singular stresses for $\xi \rightarrow \theta$ or $r \rightarrow 0$. In view of both dynamic stress solution and its all added summation terms possessing ${ }^{\mathrm{m}} \lambda_{\mathrm{i}} \geq 2(2 \leq m \leq k+1)$, e.g. is universal for static and as dynamic problems. After indicating these two stress modes as mode 
I and mode II for $\lambda_{\mathrm{i}}=0.5$, and determining the limit in Eq. (3), it ends in the following SIFs:

$$
\left.\left\{\begin{array}{l}
K_{I} \\
K_{I I}
\end{array}\right\}=\left\{\begin{array}{l}
\left.\sum_{i=\mathrm{III}} \sqrt{2 \pi L(\theta)} c_{i} \psi_{y y}(s)_{i}\right|_{\theta=0} \\
\left.\sum_{i=\mathrm{I}, \mathrm{II}} \sqrt{2 \pi L(\theta)} c_{i} \psi_{x y}(s)_{i}\right|_{\theta=0}
\end{array}\right\}=\sqrt{2 \pi L_{0}} \sum_{i=\mathrm{IIII}}\left(c_{i}\left\{\begin{array}{l}
\psi_{y y}\left(s=s_{A}\right) \\
\psi_{x y}\left(s=s_{A}\right)
\end{array}\right)_{i}\right\}\right)
$$

Where $L_{0}=L(\theta=0)$, what means the distance between the crack tip and the point $\mathrm{A}\left(\xi=1, s=s_{\mathrm{A}}\right)$ in the direction of crack surface on the boundary (fig.1) The above definition is based on the local coordinate system where the cracking direction is supposed to coincide with the global $\mathrm{x}$-axis.

For a domain involving a crack surface at an arbitrary way, the stresses should first be changed by the standard method to normal (mode I) stress $\sigma_{\mathrm{n}}$ and shear (mode II) stress $\tau_{\mathrm{n}}$ on the cracking surface plane at the point A so that Eq. (4) may be considered:

$$
\left.\left\{\begin{array}{l}
K_{I} \\
K_{I I}
\end{array}\right\}=\sqrt{2 \pi L_{0}} \sum_{i=1, I I}\left(c_{i}\left\{\begin{array}{l}
\sigma_{n}\left(s=s_{A}\right) \\
\tau_{n}\left(s=s_{A}\right)
\end{array}\right\}_{i}\right\}\right)
$$

It should be remarked that the point $\mathrm{A}$ does not need to be a prevailing node.

\section{Fast Fourier transform to quantify transient response}

Regard a periodic excitation force $p(t)$ by $N$ number of discrete values explained $p_{\mathrm{n}}=p\left(t_{\mathrm{n}}\right)=p(n \Delta t)$ where $\Delta t=T_{0} / N$ is the sampling interval, $T_{0}$ the period and $n$ extends from 0 to $\mathrm{N}-1$. The array $p_{\mathrm{n}}$ characterizing the discretized force function may be rendered being a superposition of $N$ harmonic functions

$$
p_{n}=\sum_{j=0}^{N-1} P_{j} e^{i\left(j 2 \pi f_{0} t_{n}\right)}=\sum_{j=0}^{N-1} P_{j} e^{i(2 \pi n j / N)}, \quad n=0, \ldots, N-1
$$

where $f_{0}=1 / T_{0}$ stands for frequency $(\mathrm{Hz})$ of first harmonic in the periodic extension of $p(t), f_{\mathrm{j}}=j . f_{0}$ is the frequency of the jth harmonic, and $P_{\mathrm{j}}$ is a complex-valued coefficient which determines the amplitude and period of jth harmonic. $P_{\mathrm{j}}$ can be written as

$$
P_{j}=\frac{1}{N} \sum_{j=0}^{N-1} P_{j} e^{-i\left(j 2 \pi f_{0} t_{n}\right)}=\frac{1}{N} \sum_{j=0}^{N-1} P_{j} e^{-i(2 \pi m j / N)}, \quad j=0, \ldots, N-1
$$

Eqs (6) and (7) circumscribe a discrete Fourier transform two: the array $P_{\mathrm{j}}$ is the DFT of the excitation sequence $p_{\mathrm{n}}$, and the array $p_{\mathrm{n}}$ means the inverse of DFT (IDFT) of the sequence $P_{\mathrm{j}}$. The frequency of the highest harmonic comprised in Eqs (6) and (7) is known being Nyquist frequency, introduced by $N \cdot f_{0} / 2=1 /(2 \Delta t)$.

A complex frequency-response function $H_{\mathrm{j}}$ is calculated for each $f=f_{\mathrm{j}}(0 \leq j \leq$ $N / 2$ ). Taking into account that Eq. (6) is a one-sided Fourier expansion, the values on both sides of $j=N / 2$ have to be complex conjugates of each other, that means

$$
H_{j}=H_{N-j}^{*}, \quad j=\frac{N}{2}+1, \ldots, N-1
$$


where $H^{*}$ indicates the complex conjugate of $H$. The response to each harmonic constituent of the excitation can be evaluated in the form

$$
U_{j}=H_{j} P_{j}, \quad j=0, \ldots, N-1
$$

Lastly, the response $u_{\mathrm{n}}=u\left(t_{\mathrm{n}}\right)$ at discrete time moments $t_{\mathrm{n}}=n \Delta t$ is calculated by the IDFT of $U_{\mathrm{j}}$

$$
u_{n}=\sum_{j=0}^{N-1} U_{j} e^{i\left(j 2 \pi f_{0} t_{n}\right)}=\sum_{j=0}^{N-1} U_{j} e^{i(2 \pi m j / N)}, \quad n=0, \ldots, N-1
$$

The aforesaid passage deals with the classical DFT solution to the transient elastodynamics, namely in the frequency domain. In so doing, the fast Fourier transform is a very effective and precision way of the DFT - IDFT two determination.

\section{Questions under discussion}

To prove the evolved frequency-domain technique based on the SBFEM, one standard dynamic fracture problem was simulated. Concurrently, complex frequency response functions for frequencies $f_{\mathrm{j}}(j=1, \ldots, \mathrm{M})$ are reckoned using the Frobenins solution procedure. The minimum frequency is $f_{1}=0 \mathrm{~Hz}$ (static case) and the maximum frequency is represented by the quantity $f_{\mathrm{M}}=f_{\max }$. At the same time, the damping effect is taken into consideration by adapting the elastic moduli to include an internal damping coefficient $\beta$, charactering the complex Young's modulus $E_{\mathrm{c}}=E(1+i 2 \beta)$ and the complex shear modulus $G_{\mathrm{c}}=G(1+$ $i 2 \beta)$ where $E$ and $G$ are the elastic Young's modulus and the elastic shear modulus, respectively. The dynamic stress intensity factors $K_{\mathrm{Ij}}$ and $K_{\mathrm{IIj}}(j=1, \ldots$, $M)$ are extracted next directly from the stress responses. The foregoing operation is succeeded by a FFT of the transient load Eq. 7 to get the coefficients $P_{\mathrm{j}}(j=$ $0, \ldots, N-1)$. The frequency responses $H_{\mathrm{j}}(j=0, \ldots, \mathrm{N} / 2)$ are then interpolated from the $M$ number of $K_{\mathrm{Ij}}$ and $K_{\mathrm{IIj}}$. As a rule, the interpolation is indispensable for a much greater $N$ (an integer power of 2) can be desirable to precisely meant the transient load while the complex response functions may be quite example of a smaller $M$ number of frequencies. This may put by extensively computing cost. The proper frequency-response functions are made whole by Eq. (8). Lastly, an inverse FFT Eq. (10) of $U_{\mathrm{j}}(\mathrm{j}=0, \ldots ., \mathrm{N})$ calculated by Eq. (9) is performed to obtain the response $u_{\mathrm{n}}(n=0, \ldots, \mathrm{N})$, that is, the time process of dynamic stress intensity factors. The functions $f f t()$ and ifft( ) are employed according to MATLAB to conduct FFT and IFFT, respectively. In so doing, damping coefficients of $\beta=0.0,0.001,0.01,0.025$ and 0.05 are simulated. For each $\beta$, the excitation frequencies $f_{1}=0$ to $f_{\max }=500000 \mathrm{~Hz}$ with an interval $\Delta f=1000 \mathrm{~Hz}$ are modelled, ending in $M=501$ points on a complex frequency response curve. A convergence tolerance of $\alpha=1 e-3$ is applied for all the analyses. The influences of the mesh density, the damping coefficient $\beta$, the maximum frequency $f_{\max }$ and the frequency interval $\Delta f=f_{\max } /(M-1)$ on the final exactitude and the computational cost are treated. For all the calculations, two-node linear line elements are applied. 


\section{Rectangular plate weakened by a slanting edge crack}

The said isotropic plate is subject to uniform traction on the upper surface. The geometry of the plate and boundary condition are demonstrated in fig. $2 \mathrm{a}$.
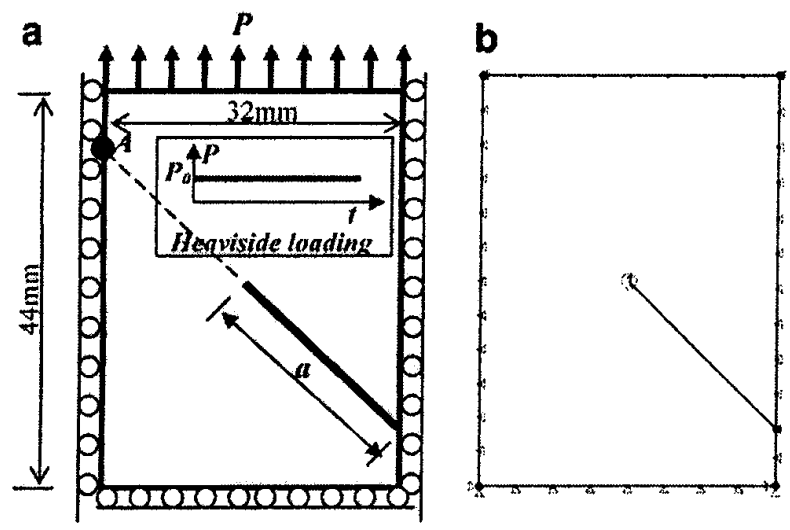

Figure 2: A rectangular plate with a slanting edge crack. (a) Dimensions, boundary and loading conditions, (b) coarse mesh including.

The material properties mean the shear modulus $G=29 \mathrm{GPa}$, Poisson's ratio $v=0.286$ and density $\rho=2450 \mathrm{~kg} / \mathrm{m}^{3}$. A mixed-mode fracture problem is the matter for which the Heaviside step loading is simulated (see fig. 2a). At the same time, all SIFs are normalized by $P_{0}(\pi \mathrm{a})^{1 / 2}$ where $P_{0}$ stands for the quantity of the transient loading and $a=23 \mathrm{~mm}$ is the crack length, when supposing a plane strain condition. One subdomain is introduced possessing the scaling centre at the crack tip. One mesh with 41 nodes is indicated in fig. $2 \mathrm{~b}$. The stresses at the point A in fig (2.a) are used to extract SIFs in compliance with Eq. (5).

Examples of the calculated DSIFs are demonstrated in figs. 3 and 4 where the time histories of $K_{\mathrm{I}}$ and $K_{\mathrm{II}}$ are shown, computed employing the course mesh with varied material damping coefficients $\beta$ compared to the issues obtained in by Fedelinski et al. using the BEM.

There is very fair material harmony applying $\beta=0.01$ for both $K_{\mathrm{I}}$ and $K_{\mathrm{II}}$. The coarse mesh and the fine mesh results in almost identical outcomes demonstrating that the course mesh with only 41 nodes is fine plenty (see fig. 4). The same isotropic problem with a little various material properties was simulated by Song [2] applying the time-domain SBFEM and a finer mesh with 23 super-elements, and over 200 nodes. This expresses one of the disadvantages of time-domain methods, that means a mass matrix has to be applied in timeintegration. Since the mass matrix used in [2] is the low-frequency expansion of the dynamic stiffness matrix, it may just symbolize the inertial results at low frequencies. The higher frequency constituents have to be an example of fine meshes with small dimensions. The frequency-domain method, on the other 


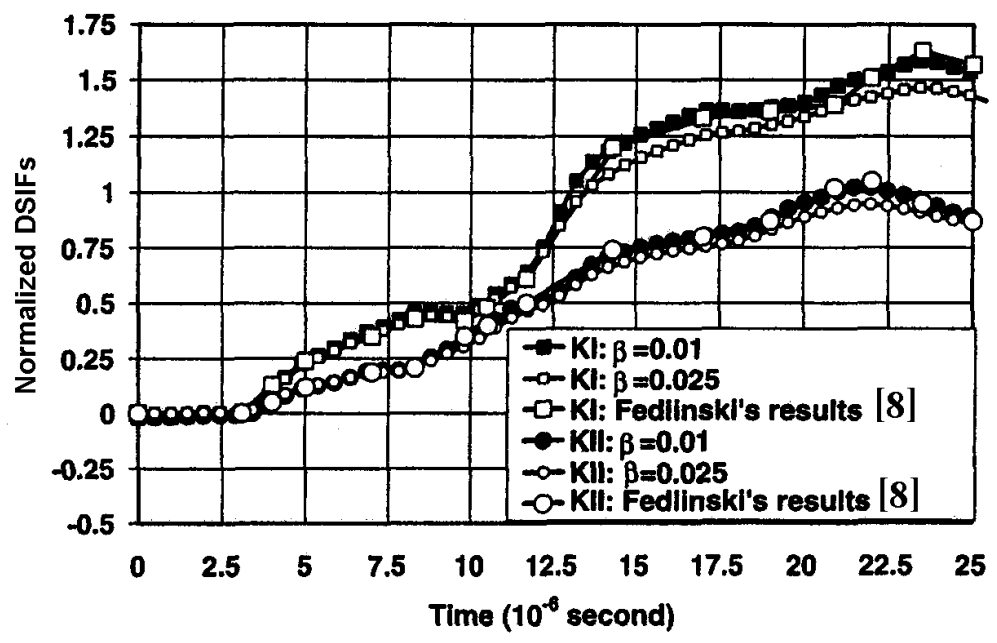

Figure 3: Normalized DSIFs applying the coarse mesh: influence of material damping coefficient $\beta$ (taken from [5]).

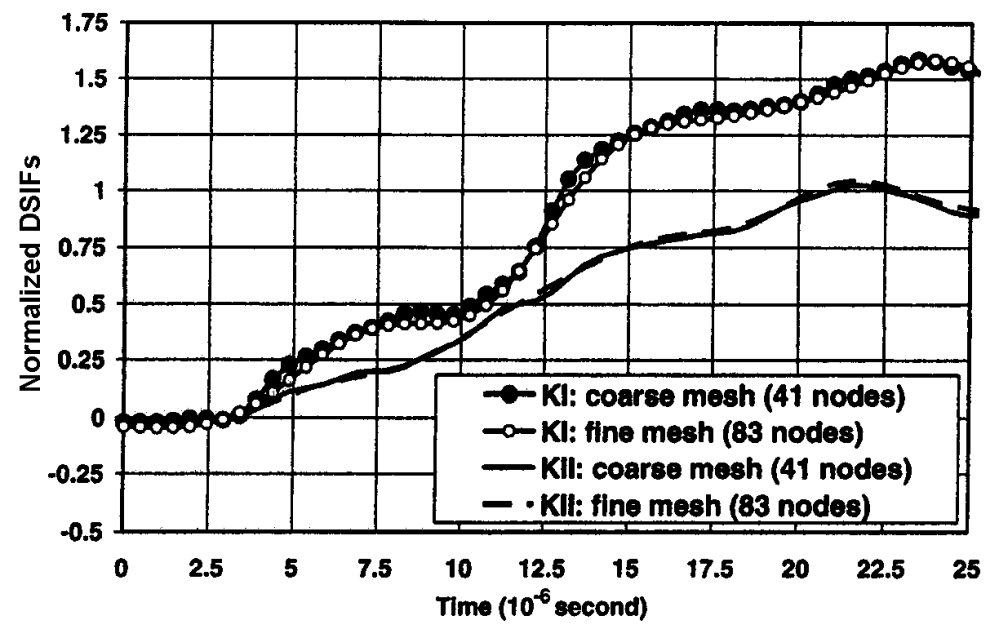

Figure 4: $\quad$ Normalized DSIFs using $\beta=0.01$ : influence of mesh density.

hand, does not require mass matrices. This permits precise computation of time responses applying coarse meshes like this as fig. 2(b). The orthotropic instance of the same plate (see fig. 2.(a)) in plane stress is modelled applying the identical mesh (fig. 2(b)). The properties in the principal material axes stand for: Young's modulus $E_{1}=82 \mathrm{GPa}, E_{2}=\mathrm{R} \cdot \mathrm{E}_{1}$, shear modulus $G_{12}=29 \mathrm{GPa}$, Poisson's ratio $v_{12}=0.4006$ and the mass density $\rho=2450 \mathrm{~kg} / \mathrm{m}^{3}$. The same problem was solved by Song applying the time-domain SBFEM [2] and Albuquerque et al. using the BEM [7]. Two events of $R=0.5$ and 2.0 are studied. 


\section{Methodology of simulations and remeshing routine}

A domain can be advantageously divided into subdomains in the SBFEM just as it can be stood for by elements in the FEM. On account of the semi-analytical essence of the SBFEM, the dimension and shape of subdomains in a region can be altered to a great degree if desired without relinquishment of the resulting exactitude. As well, the scaling center can be put at the required place within the subdomain (if the domain boundary is observable from the scaling center). The nodal density in various subdomains can be very altered, too. A numerical model of LEFM - based mixed-mode crack growth applying the SBFEM presupposes the phases:

1. Subdivide the initial domain into some subdomains by straight lines or edges (see fig. 5) in view of the displacement constraints and loading conditions. The topological information of the domain is fully represented by the edges and their intersections, i.e., vertices.

Each edge is related with two vertices. Different seeds can be specified to different edges or subdomains so that the nodal density from edge to edge or from subdomain to subdomain can be flexibly varied as required. The loading conditions and boundary constrains are also related to edges: Each subdomain is fully represented by the connected vertices placed in a line counterclockwise round the scaling centre. Fig. 5 shows that a domain is composed of four subdomains with the subdomain 4 (S4) being the crack subdomain. Placing scaling centers for the normal subdomains can be optimized by taking advantage of non-discretization of unrestricted side-front surfaces.

Namely, we get the crack growth direction $\theta_{0}$ from the conditions

$$
\begin{aligned}
& G(\theta)=\frac{4}{E}\left(\frac{1}{3+\cos ^{2} \theta}\right)^{2}\left(\frac{1+\frac{\theta}{\pi}}{1-\frac{\theta}{\pi}}\right)^{-\frac{\theta}{\pi}}\left[\left(1+3 \cos ^{2} \theta\right) K_{I}^{2}-8 \sin \theta \cos \theta K_{I} K_{I I}\right]+ \\
& \left.\begin{array}{rl}
\frac{\partial G(\theta)}{\partial \theta}=0, \text { and } \frac{\partial^{2} G(\theta)}{\partial \theta^{2}}<0 & \left.+\left(9-5 \cos ^{2} \theta\right) K_{I I}^{2}\right]
\end{array}\right\}
\end{aligned}
$$

For the maximum stress energy release rate it holds $G_{\max }=G(\theta)$. The scaling factor is equal to

$$
\alpha=\sqrt{\frac{G_{I C}}{G_{\max }}}
$$

where $G_{\text {IC }}$ is the critical SERR or else fracture energy being interrelated to the material fracture toughness $K_{\mathrm{IC}}$ in the form

$$
G_{I C}=\frac{K_{I C}^{2}}{E} \text { for plane stress and } G_{I C}=\frac{\left(1-v^{2}\right) K_{I C}^{2}}{E} \text { for plane strain }
$$




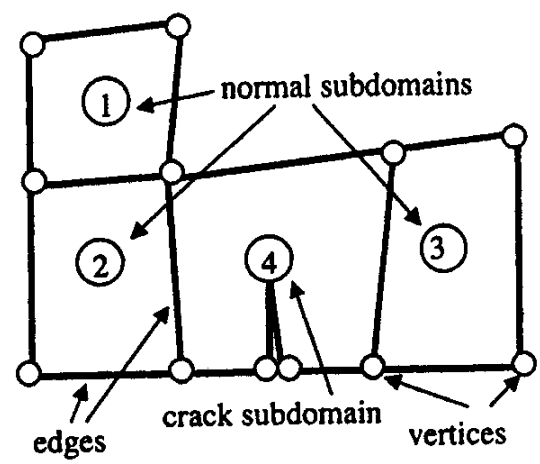

Figure 5: Subdomains.

Scaling the reference external loading vector and displacement reactions on crack growth is performed; it follows that

$$
\begin{gathered}
p_{\mathrm{s}}=\alpha p_{0} \\
u_{\mathrm{s}}=\alpha u_{0}
\end{gathered}
$$

where $p_{\mathrm{s}}$ is the scaled loading vector minimal for the crack to growth and $u_{\mathrm{s}}$ means the displacement field of the domain subject to $p_{\mathrm{s}}$, with the stress field recovered in the region.

The easy remeshing routine permits fully automatic crack growth modelling to be attained without user interferences. Improvements may lead to more sophisticated remeshing procedures but the current one seems sufficient for numerical examples. The smoothness of modelled crack trajectories depends on the pre-determined crack addition length.

\section{Conclusion}

Investigations employing the developed frequency-domain way of tackling have been performed on two standard problems with both isotropic and orthotropic material characteristics. Numerical issues indicate that this approach is competent of calculating time processes of DSIFs properly and strikingly involving a small number of degrees of freedom. It is expected when combined with the simple remeshing in the procedure SBFEM, this frequency-domain way will provide a very competitive system for simulating dynamic crack growth tasks. As well, it is learned that calculating the complex frequency-response functions is considerably time-consuming than FFT and IFFT.

The maximum frequency and the frequency interval that are important factors for the complex frequency-response functions to be applied in IFFT, should be chosen suitably. Internal material damping is considered to influence numerical both outcomes and stability.

The newly-evolved SBFEM was used to simulate the LEFM-based mixedmode crack growth in brittle and quasi-brittle materials. 


\section{Acknowledgements}

The author gratefully acknowledges the financial support of the presented research by the Grant Agency of the Czech Republic (project No. 103/06/1382) and Grant Agency of the Academy of Sciences of the Czech Republic (project IAA 200710604).

\section{References}

[1] Ariza, M.P., Dominguez, J., General BE approach for three-dimensional dynamic fracture analysis. Engng Anal Bound Elem, 26, pp. 639-651, 2002.

[2] Song, C.M., A super-element for crack analysis in the time domain. Int $J$. Numer Meth Engng, 61, pp. 1332-1357, 2004.

[3] Wolf, J.P., Song C.M., Finite-element modelling of unbounded media. Chichester: John Wiley and Sons, 1996.

[4] Wolf J.P., The scaled boundary finite element method. Chichester: John Wiley and Sons, 2003.

[5] Yang, Z.J., Deeks, A.J., HaO, H.A., Transient dynamic fracture analysis using scaled boundary finite element method: a frequency-domain approach. Engineering Fracture Mechanics, 74, pp. 669-687, 2007.

[6] Yang, Z.J., Fully automatic modelling of mixed-mode crack propagation using scaled boundary finite element method. Engineering Fracture Mechanics, 73, pp. 1711-1731, 2006.

[7] Albuquerque, E.L., Sollero, P., Aliabadi, M.H., The boundary element method applied to time dependent problems in anisotropic materials. International Journal of Solids and Structures, 39, pp. 1405-1422, 2002. 